\title{
Determinantes e impacto de los sentimientos de autoeficacia en los profesores
}

\section{Carmen Gloria Covarrubias-Apablaza}

Universidad de Talca, Chile cacovarrubias@utalca.cl

\section{Michelle Celinda Mendoza-Lira}

Universidad Autónoma de Chile, Chile mmendozal@uautonoma.cl

\section{Resumen}

Los centros educativos, en la actualidad, deben enfocar sus prácticas de enseñanza hacia el fomento del desarrollo intelectual, y de la motivación intrínseca en los estudiantes, con tal de que adquieran las competencias para aprender durante toda la vida. Los sentimientos de autoeficacia constituyen una fuerza poderosa que incide en el campo conductual, actitudinal y relacional de profesores y alumnos. Este artículo tiene el propósito de exponer los resultados de los sentimientos de autoeficacia en una muestra de 544 profesores chilenos según el nivel de enseñanza (infantil, primario y secundario) y la administración del centro (público, particular subvencionado y particular pagado). Los hallazgos de este estudio revelan diferencias significativas en los sentimientos de autoeficacia docente según el tipo de administración del centro educativo, pero no asi en los niveles de enseñanza.

\section{Palabras clave}

Gestión escolar; sistema educativo chileno; formación de profesores; calidad de la enseñanza; Chile (Fuente: Tesauro de la Unesco).

\footnotetext{
Recepción: 2015-10-13 | Envío a pares: 2016-07-16 | Aceptación por pares: 2016-08-05 | Aprobación: 2016-08-30 


\title{
The Determining Factors and Impact of Self-Efficacy Feelings on Teachers
}

\begin{abstract}
Nowadays, educational centers must guide their teaching practices towards promoting intellectual development and the intrinsic motivation of students, as long as they acquire the skills to learn throughout their entire life. Self-efficacy feelings are a strong force that affects the behavioral, attitudinal and relational fields of both teachers and students. This article aims to show the results of self-efficacy feelings on a sample consisting of 544 Chilean teachers based on teaching level (nursery, elementary and high school) and management of the educational center (public, subsided private and paid private). The article's findings reveal significant differences in the selfefficacy feelings of teachers based on the type of management of the educational center, but not in teaching levels.
\end{abstract}

\section{Keywords}

School Management; Chilean Educational System; Teacher's Training; Teaching Quality; Chile (Source: Unesco Thesaurus). 


\section{Determinantes e impacto dos sentimentos de autoeficácia nos professores}

Resumo

Na atualidade, os estabelecimentos de ensino devem focalizar suas práticas de ensino para o fomento do desenvolvimento intelectual, e da motivação intrínseca nos estudantes, com o objetivo de eles adquirirem as competências para a aprendizagem ao longo de toda a vida. Os sentimentos de autoeficácia constituem uma força poderosa que incide no âmbito comportamental, atitudinal e relacional de professores e alunos. Este artigo tem o propósito de expor os resultados dos sentimentos de autoeficácia, em uma mostra de 544 professores chilenos segundo o nivel de ensino (infantil, fundamental e médio), e a administração do estabelecimento (público, particular subsidiado e particular pago). Os achados deste estudo revelam diferenças significativas nos sentimentos de autoeficácia docente segundo o tipo de administração do estabelecimento educativo, mas não nos níveis de ensino.

\section{Palavras-chave}

Gestão escolar; sistema educativo chileno; formação de professores; qualidade do ensino; Chile (Fonte: Tesauro da Unesco). 


\section{Teoría de autoeficacia}

La teoría de autoeficacia ha pasado por distintas etapas conceptuales y metodológicas. Desde sus comienzos hasta la actualidad, esta teoría ha intentado demostrar cómo los aspectos cognitivos, conductuales, contextuales y afectivos de las personas están condicionados por la autoeficacia. Albert Bandura (1977), su máximo impulsor, preocupado por elevar a ciencia la psicología y validar sus postulados, buscó un método eficaz que resolviera problemas humanos.

Es así como el constructo de autoeficacia tomó la forma y la consistencia suficiente para propiciar la creación de una teoría cuyo eje central, el pensamiento autorreferente, actúa mediando la conducta y la motivación en las personas (Bandura, 1987). No obstante, para que el pensamiento autorreferente sea convergente y coherente con el pensar, sentir y actuar, requiere de la calidad del autoconocimiento y la opinión de eficacia personal que se tenga. De ahí que cambio y adaptación serán las consignas del funcionamiento humano bajo la perspectiva sociocognitiva; donde la capacidad de autorreflexión y de autorregulación dependen de los factores conductuales, personales y ambientales.

Por otro lado, cabe resaltar que en los factores personales, conductuales y ambientales se produce una interrelación e interdependencia que, a su vez, conforma una tríada de determinación recíproca. Esta reciprocidad influye intencionadamente en el funcionamiento psicosocial y en los acontecimientos que afectan la vida de las personas, ya que al luchar por el control de sus vidas intentan obtener ganancias sociales y personales. Por ello, el resultado de sus acciones está condicionado por los pensamientos que tienen de sí mismas, pues, al ser productoras y productos de sus circunstancias, no solo responden a las demandas del ambiente, sino que, además, las modifican (Bandura, 2004).

Bandura (2008) manifiesta que las personas no tienen un funcionamiento autónomo, ni tampoco sus comportamientos están totalmente determinados por factores situacionales. Las personas son producto de la interacción recíproca entre los determinantes personales, comportamentales y ambientales. Así, en lo personal y comportamental, están influidas de forma directa por la dotación biológica, como por las propias concepciones, valores, metas y estados afectivos. Lo ambiental y lo comportamental alteran el entorno y viceversa. Mientras que lo personal y lo ambiental están intervenidos por los modelos, las influencias sociales o la educación, entre otros, que modifican los atributos personales.

Otro aspecto interesante propuesto por Bandura $(1977,1987,1999)$ ha sido la diferenciación sostenida entre expectativas de eficacia y expectativas de resultados. Las primeras han sido definidas como el convencimiento de que una ejecución se realice con éxito y, las segundas, como la estimación de los resultados que se deben conseguir una vez realizada la ejecución. Así, mientras unas están referidas al convencimiento de hacer bien una ejecución, las otras están referidas a lo esperado por la ejecución. Para Bandura, los comportamientos son determinantes de los resultados, que a su vez dependen de la gestión de los propios recursos.

No obstante, Bandura (1987) consciente de que el poder de una teoría psicológica reside en la capacidad de definición, predicción y descubrimiento de factores que regulan interrelacionadamente la conducta humana, propuso mecanismos, fuentes y procesos implicados en la autoeficacia.

Los sentimientos de autoeficacia operan según los siguientes mecanismos: la selección de conductas, el esfuerzo y la persistencia, los pensamientos y las reacciones emocionales, así como la producción y predicción de la conducta. Con respecto a lo antes mencionado, las diversas circunstancias de la vida incitan a las personas a tomar decisiones o a realizar actuaciones destinadas al logro de los propósitos que se han planteado. Asimismo, albergan las facultades para poder enfrentar obstáculos o superar las 
circunstancias adversas con un efecto distinto sobre las que poseen altos o bajos sentimientos de autoeficacia, siendo determinante para gestionar, predecir e influir en los acontecimientos de los distintos ámbitos de vida.

Aún más, el sentimiento de autoeficacia puede ser desarrollado a través de cuatro fuentes: las experiencias de dominio, las experiencias vicarias, la persuasión social y las experiencias afectivas. Las primeras constituyen experiencias de dominio real con una enorme implicación en la fortaleza o debilidad de las autopercepciones de eficacia. Las segundas están referidas al modelado, que es la observación de ejecuciones realizadas por terceros. Las terceras consisten en convencer al sujeto que tiene las capacidades necesarias para lograr lo que se ha propuesto. Y las últimas afectan los sentimientos de autoeficacia mediante los estados psicológicos y emocionales, positivos o negativos que tengan las personas.

Junto a los mecanismos que operan y a las fuentes de las cuales se nutre, la autoeficacia también tiene la capacidad de activar procesos cognitivos, motivacionales, afectivos y selectivos. Así es como activando la cognición, las personas pueden predecir y regular los sucesos que les acontecen, donde la motivación juega un papel fundamental en la diferenciación y valoración de lo que pueden y no pueden hacer. Sumado a ello, los procesos afectivos posibilitan la identificación de las amenazas potenciales del entorno, para seleccionar las conductas más adecuadas en la consecución de sus propósitos.

Por último, el impacto de lo antes dicho marca profundas diferencias en las personas con un fuerte o un débil sentimiento de autoeficacia. Las primeras poseen un interés intrínseco que las hace esforzarse y perseverar pese a las dificultades; son capaces de visualizar los problemas como desafíos, oportunidades de aprendizaje y, por ende, de progreso en sus habilidades. Por su parte, las segundas carecen de compromiso; flaquean rápidamente ante las adversidades; evitan tareas complejas; huyen de los problemas y dejan de creer en sí mismas.

\section{Autoeficacia en profesores}

En consideración de lo antes dicho, las complejas demandas emanadas de la escuela, la política y la sociedad, impactan fuertemente en los sentimientos de autoeficacia y en las capacidades del profesorado para suscitar el aprendizaje en los estudiantes, y mantener el compromiso con su profesión (Klassen, Tze, Betts y Gordon, 2011). Conforme a ello, la cantidad de estudios que han intentado explicar la realidad educativa por medio de la autoeficacia abarca casi tres décadas, valorando el importante papel que tiene en la conducta humana y, por ende, en la práctica pedagógica (Bandura, 1987; Pajares, 2006).

Como consecuencia de lo anterior, la investigación sobre la opinión de eficacia personal en contextos educativos ha sido bastante prominente ( $\mathrm{Pa}-$ jares, 2006). En términos concretos, los estudios de autoeficacia han marcado una destacada trayectoria por su amplio desarrollo teórico y empírico (Blanco, 2009). De hecho, se constata un aumento gradual desde 1998 a 2009 de la investigación en torno a la eficacia del profesor, con diversos enfoques metodológicos, dominios específicos e internacionalización del constructo, como recogen Klassen et al. (2011).

Para Albert Bandura, la eficacia del profesor constituye un tipo de autoeficacia (Prieto, 2007) que contempla una evaluación de las propias capacidades para alcanzar un nivel adecuado de rendimiento en una tarea y entorno específico. Pero, para alcanzar un desempeño óptimo, los docentes deben manejarse en dos niveles de pensamiento, por un lado, sentirse capaces y, por otro, juzgarse capaces. La combinación de estos dos niveles indica conocimiento, despliegue y gestión de las competencias, habilidades o capacidades que poseen, de manera que el sentimiento de autoeficacia actúa como un mecanismo cognitivo mediador, entre sus conocimientos y sus actos pedagógicos (Bandura, 1999).

En otras palabras, la confianza de los profesores en lo que saben y hacen diariamente en los contextos educativos es de gran trascendencia para 
que los procesos educativos aseguren la calidad de la enseñanza y el logro del aprendizaje en los estudiantes. Los profesores que son dueños de una alta confianza y opinión de eficacia personal, se mostrarán preocupados por suscitar el aprendizaje en los estudiantes, y esperarán lo mejor de ellos. Al contrario, profesores que dudan de sus capacidades, y están inundados por representaciones negativas del proceso educativo y de sus estudiantes, socavarán la motivación intrínseca y extrínsecas de los últimos (Pintrich y Schunk, 2006).

La necesidad de capturar un constructo tan complejo y esquivo como es la autoeficacia del profesor ha suscitado el interés de su estudio tanto en materia teórica como metodológica. Durante las etapas iniciales, la incipiente conceptualización no tenía límites claros entre lo que aportaban Rotter o Bandura; esta confusión acarreó serias dificultades para comprender y medir el constructo (Prieto, 2007). Las dificultades permanecieron hasta la década de los noventa, con la diferencia de que la tendencia investigativa se amparó en los postulados de Bandura, y en la preocupación por la construcción de medidas válidas y fiables, que no han estado, sin embargo, exentas de críticas (Charalambous, Philippou y Kyriakides, 2008; Ciani, Summers y Easter, 2008).

Los estudios iniciales consideraban que la eficacia docente constituía una variable explicativa de gran alcance, puesto que surtía efectos positivos en el porcentaje de objetivos logrados, en el rendimiento de los estudiantes, además del cambio y el compromiso que asumían los profesores frente a las demandas de la política educativa (Armor et al., 1976; Berman et al., 1977). En esta primera etapa de conceptualización, el constructo era abordado como la capacidad para la obtención de resultados positivos en el aprendizaje de los estudiantes. Sumado a ello, esta concepción incipiente recibió el influjo del locus de control, donde los profesores asumían la responsabilidad por aquellos factores que estaban a su alcance.
De manera similar, pero con tintes de cambio, Gibson y Dembo (1984) reconciliaron los esfuerzos de Rotter y Bandura. Para ello, examinaron la propuesta de RAND, y ofrecieron una nueva que consistía en que el lugar de control interno y externo estaban relacionados con las expectativas de autoeficacia y las expectativas de resultado. Con el objetivo de dar más consistencia a su propuesta, sugirieron la existencia de dos tipos de autoeficacia en el profesor: una general (expectativas de resultados), y otra personal (expectativas de autoeficacia). La autoeficacia docente general la concebían como la capacidad del docente para promover cambios en los estudiantes, encontrándose limitada por factores externos que escapan a su control. En cambio, la autoeficacia docente personal -más cercana a los postulados de Bandura- la entendían como el convencimiento que tienen los profesores de sus destrezas y habilidades para favorecer el aprendizaje en los estudiantes.

A modo complementario, es importante destacar que estos primeros intentos por proporcionar un marco conceptual al constructo del sentimiento de autoeficacia del profesor, no solo fueron espejo para las ideas de Rotter y Bandura, sino que además, constituyeron el reflejo del paradigma educativo de la época: proceso-producto. Por este motivo, la conceptualización de la eficacia del profesorado estaba orientada al tipo de causalidad y a las capacidades pedagógicas para influir en el rendimiento de los estudiantes.

Años más tarde (década de los noventa), con mayor claridad, la eficacia del profesor recibió nuevas influencias. Las investigaciones de la época concibieron este sentimiento como la capacidad para influir en el aprendizaje de los alumnos, a pesar de las dificultades cognitivas, sociales o comportamentales que pudiesen presentar. Claramente, hay un vuelco en la mirada de las prácticas pedagógicas y los resultados de los estudiantes; los profesores deben invertir un mayor esfuerzo y persistir tenazmente, aun 
cuando los estudiantes estén desmotivados, pertenezcan a entornos socioculturales desfavorecidos o tengan discapacidad intelectual (Coladarci y Breton, 1997; Guskey y Passaro, 1994; Soodak y Podell, 1996).

En convergencia con las definiciones de la época, Tschannen-Moran et al. (1998) contribuyeron significativamente al constructo de autoeficacia docente definiendo la eficacia del profesor como la capacidad para promover la participación estudiantil y el aprendizaje, pese a la desmotivación y los problemas conductuales que presenten los estudiantes. Resulta evidente que dicha conceptualización recoge el paradigma del aprendizaje autónomo, al considerar que el rendimiento del estudiante no es un resultado final, sino el proceso de un aprendizaje, en el cual los estudiantes se transforman en sujetos activos del proceso educativo.

No obstante, con el fin de configurar un constructo cada vez más específico, Tschannen-Moran y Woolfolk (2001) proponen una conceptualización enfocada más en las capacidades de que dispone el profesor a fin de organizar y ejecutar cursos de acción necesarios para lograr con éxito una tarea de enseñanza específica en un contexto particular. Esta concepción refleja en mayor medida la acción del docente eficaz, al representar un juicio de las capacidades para enseñar y suscitar aprendizaje en determinadas y variadas circunstancias (Prieto, 2007).

Aportes recientes surgen de la opinión de Ross y Bruce (2007) que conciben la eficacia del profesor como una expectativa que promueve el aprendizaje en los estudiantes. También otros consideran que la autoeficacia del profesor es la capacidad para realizar con éxito tareas específicas de enseñanza (Dellinger, Bobbet, Olivier y Ellet, 2008). Mientras, Pas, Bradshaw y Hershfeldt (2012) opinan que este sentimiento está referido a la capacidad de los profesores para crear un ambiente adecuado para el aprendizaje.

Para complementar lo que se entiende por autoeficacia en el profesor, es imprescindible tener en cuenta aquellos elementos o factores asociados a este sentimiento. Según De la Torre y Casanova (2007) la eficacia en la enseñanza afecta a: el pensamiento; la toma de decisiones y el comportamiento. Por esto, aquellos profesores que tienen un adecuado nivel de eficacia movilizarán todas las acciones, para que sus estudiantes aprendan y logren buenos resultados. Además, utilizarán estrategias de enseñanza variadas, en consonancia con las necesidades que tengan sus alumnos. Incluso serán más proclives a reflexionar sobre sus prácticas y desempeños pedagógicos.

Ahora bien, es importante recordar que el sentimiento de autoeficacia en el profesorado también se ve influenciado por el contexto del aula, la escuela y el entorno; además, por los estudiantes, los colegas, la dirección y la comunidad. Los profesores son líderes de un escenario de aprendizaje y a la vez empleados de una organización, ejecutando sus quehaceres pedagógicos cotidianos, socializando con el resto de la organización y contribuyendo con la sociedad (Bueno, 2004; Friedman y Kass, 2002). Por ello su eficacia personal debe ser una muestra clara de que tienen las capacidades para ejecutar sus tareas con lo mejor de sus competencias cognitivas, afectivas y actitudinales.

En una educación de calidad no basta con que los profesores tengan amplios conocimientos y habilidades necesarias para el cargo, sino que, además, deben ser poseedores de una férrea confianza o convicción en lo que hacen. Para Rizvi y Elliot (2005), el sentimiento de autoeficacia del profesorado es parte sustancial de su profesionalidad, así como también lo son la práctica, el liderazgo y la colaboración. Un funcionamiento pedagógico eficaz para alcanzar un alto grado de destreza requiere el desarrollo de dos procesos: el crecer y el aprender. En la medida que estén articulados cada uno de los elementos mencionados, los profesores podrán diseñar y organizar ambientes de enseñanza y aprendizaje eficaces (Philippou y Christou, 2002). 


\section{Autoeficacia en profesores según el nivel de enseñanza y el tipo de administración del centro}

El nivel de enseñanza donde imparten docencia los profesores también presenta influencias importantes en sus sentimientos de eficacia. Los estudios indican que los profesores de educación primaria tienen una mejor opinión de eficacia que los de secundaria; opinión mediada por las mayores expectativas de rendimiento positivo en sus estudiantes, una mayor satisfacción profesional y, en cierta medida, por un mayor control del comportamiento en el aula. De igual forma, los informes de una menor eficacia en los profesores de secundaria podrían explicarse por el tipo de asignatura que enseñan, los cursos asignados, los periodos del año, entre otros (Tschannen-Moran y Woolfolk, 2001, 2007).

Cabe destacar que esta variable también presenta diferencias en la eficacia del profesorado para promover la participación y el compromiso en los estudiantes, que sería mayor en los de primaria, que en los de secundaria. Una explicación de esto podría estar en la dificultad que presentan los adolescentes, en comparación con los más pequeños, para implicarse en el aprendizaje. Además, los estudiantes de cursos inferiores, al compartir más tiempo con sus profesores, entregan información adicional y más detallada de sus necesidades reales. Esto último permite que las rutinas de enseñanza, no solo motiven el aprendizaje, sino que también aumenten los juicios de eficacia personal del profesorado (Guo, Justice, Sawyer y Tompkins, 2011; Wolters y Daugherty, 2007).

A pesar de que Soodak y Podell (1997) encuentran cierta homogeneidad en los niveles de eficacia entre profesores de primaria y secundaria, proponen una serie de explicaciones en cuanto a las semejanzas o diferencias que podrían arrojar las muestras: a) constituyen muestras distintas con enfoques educativos y alumnos distintos; b) el profesorado alberga motivaciones diferentes para estudiar pedagogía (los primeros podrían basar su selección en las temáticas propias de la educación, mientras que los segundos se enfocarían más en una disciplina específica), y c) los contextos organizacionales (más pequeños en la educación primaria y más diversificados en la educación secundaria), marcan diferencias en las relaciones interpersonales para que sean más o menos personalizadas.

Aún cuando no hay acuerdo en cuanto al impacto que tiene la clase social en la eficacia docente, recientes publicaciones dan cuenta del impacto significativo de las características socioeconómicas del alumno y de la escuela, en las percepciones de eficacia docente (Cheung, 2006; Chong et al., 2010; Goddard, 2001).

Ahora bien, es necesario mencionar que los estereotipos socioculturales también intervienen en el análisis de la tarea que hace el profesor, que muchas veces puede estar influenciada por los medios de comunicación o por las profecías autocumplidas (Siwatu, 2011).

\section{Metodología}

\section{Objetivos}

El objetivo general de esta investigación es identificar el sentimiento de autoeficacia en una muestra de profesores en Chile. Dicho objetivo se desglosa y concreta en los siguientes objetivos específicos: a) describir los sentimientos de autoeficacia en profesores chilenos en función del nivel de enseñanza y tipo de administración del centro; b) comparar los sentimientos de autoeficacia en profesores chilenos en función del nivel de enseñanza, y c) comparar los sentimientos de autoeficacia en profesores chilenos en función del tipo de administración del centro.

\section{Hipótesis}

- Los sentimientos de autoeficacia de los profesores chilenos presentan diferencias según el nivel de enseñanza. 
- Los sentimientos de autoeficacia de los profesores chilenos presentan diferencias según el tipo de administración del centro.

\section{Diseño}

La investigación realizada ha empleado una metodología cuantitativa no experimental, de tipología descriptiva, transeccional y psicométrica (Hernández, Fernández y Baptista, 2010).

\section{Muestra}

La muestra, compuesta por 544 profesores de las áreas urbanas de las Provincias de: MargaMarga, Ouillota y Valparaíso (Chile), ha sido de tipo incidental, no probabilística. La participación de los sujetos ha sido voluntaria, encontrándose todos ellos en situación laboral activa y en posesión de la correspondiente titulación (tabla 1).

Tabla 1. Características de la muestra

\begin{tabular}{|c|c|c|c|c|c|}
\hline Variables & & & & & \\
\hline \multirow{2}{*}{ Género } & Femenino & Masculino & & & \\
\hline & $70,2 \%$ & $29,8 \%$ & & & \\
\hline $\begin{array}{c}\text { Nivel } \\
\text { Enseñanza }\end{array}$ & Infantil & Primaria & Secundaria & & \\
\cline { 2 - 7 } & $9,2 \%$ & $52,2 \%$ & $38,6 \%$ & & \\
\hline $\begin{array}{c}\text { Titularidad } \\
\text { Centro }\end{array}$ & Público & Concertado & Privado & & \\
\cline { 2 - 7 } & $40,4 \%$ & $48,2 \%$ & $11,4 \%$ & & \\
\hline $\begin{array}{c}\text { Experiencia } \\
\text { (años) }\end{array}$ & $0-5$ & $6-10$ & $11-15$ & $16-20$ & +20 \\
\cline { 2 - 7 } & $20,4 \%$ & $15,4 \%$ & $12,9 \%$ & $8,8 \%$ & $42,5 \%$ \\
\hline
\end{tabular}

\section{Procedimiento}

Para acceder a los centros educativos se contactó con los administradores de los centros, a fin de solicitar la autorización para llevar a cabo la investigación. Una vez conseguidas las correspondientes autorizaciones se procedió a enviar a los profesores una carta de presentación y el cuestionario que debían cumplimentar.

\section{Instrumento}

El instrumento de medida seleccionado que mejor se adaptaba a las necesidades de esta inves- tigación fue el Teachers Self Efficacy Scale (TSES) de Tschannen-Moran y Woolfolk (2001), en su versión larga. El instrumento consta de 24 items, puntuables en una escala de 1 a 9 ( 1 = "nada", 9 = "mucho"), que se dividen en 3 subescalas: a) "Eficacia para la implicación de los estudiantes", b) "Eficacia en las estrategias de enseñanza" y c) "Eficacia en el manejo de la clase". Posteriormente, se llevó a cabo el proceso de traducción y adaptación del TSES a lengua española. El procedimiento utilizado para la traducción de la medida del inglés al español fue el de traducción inversa o back translation (Martínez, Moreno y Muñiz, 2005).

Para validar el instrumento, 262 profesores de diferentes centros educativos, públicos, concertados y privados de las municipalidades de Valparaíso y Viña (Chile) respondieron el cuestionario con una escala tipo Likert simplificada de 1 (nada) a 5 (mucho). El cambio de escala responde a las necesidades del contexto esgrimidas por los expertos y por los participantes de una prueba piloto previa de comprensión, que consideraron que una escala con más opciones podría ocasionar confusiones.

Las técnicas estadísticas seleccionadas, que mejor se ajustaban a los datos para llevar a cabo la validación de constructo, han sido el análisis factorial exploratorio y el índice de consistencia interna alfa de Cronbach. La primera técnica estadística reveló la presencia de un cuarto factor denominado "Eficacia en la atención a la singularidad de los estudiantes" (factor D), mientras que la segunda técnica arrojó para el instrumento en general un índice de consistencia interna de 0,92. Pese a que los resultados no coinciden con la solución original del instrumento (3 factores con 24 ítems; 4 factores con 17 ítems) es posible destacar el 65,09\% porcentaje de varianza total explicada y el logro de un alfa bastante alto, lo que hace que el instrumento sea válido y fiable.

\section{Resultados}

A fin de dar respuesta al objetivo sobre la descripción de los sentimientos de autoeficacia en los 
ISSN 0123-1294 | e-ISSN 2027-5358 | Educ.Educ. Vol. 19. No. 3 | Septiembre-Diciembre de 2016 | pp. 339-354.

Universidad de La Sabana | Facultad de Educación

profesores chilenos, se consideraron las medidas descriptivas de las puntuaciones globales así como los resultados de los cuatro factores del instrumento Cabe señalar que dichos resultados, expuestos en las tabla 2 y 3 , son bastantes similares, salvo excepciones, en las variables estudiadas.

La puntación global y las puntuaciones para cada factor del cuestionario, plasmados en la tabla 2 , señalan mayores sentimientos de autoeficacia en los profesores que se desempeñan en centros privados, y menores sentimientos de autoeficacia para los profesores que imparten enseñanza en centros públicos. Además, conviene subrayar que las puntuaciones del factor $D$ revelan promedios iguales en la atención a la singularidad de los estudiantes tanto en los profesores de centros públicos como en los profesores de centros concertados.

Las medidas descriptivas del sentimiento de autoeficacia en los profesores de Chile, según el nivel educativo en el cual se desempeñan, mostradas en la tabla 3, muestran que la distribución de la puntuación global y para los factores del instrumento, es bastante homogénea en los niveles educativos infantil, primario y secundario. Sin embargo, es necesario recalcar que un análisis más detallado revela que los niveles de autoeficacia manifestados por los profesores en el factor $D$ son más altos en aquellos que imparten enseñanza en el nivel infantil. A su vez, habría que indicar que los profesores de enseñanza infantil manifiestan tener mayores sentimientos de autoeficacia para atender la singularidad de los estudiantes.

Con la finalidad de dar respuestas a los objetivos enfocados en la comparación de los sentimientos de autoeficacia de los profesores de Chile según los niveles educativos, y la titularidad de los centros, se aplicaron técnicas estadísticas paramétricas para la comparación de medias. Estas comparaciones de medias se hicieron tanto en términos globales como específicos de los factores que componen el cuestionario aplicado.

Tabla 2. Estadísticos descriptivos del sentimiento de autoeficacia de los profesores para cada factor, según la titularidad del centro

\begin{tabular}{|c|c|c|c|c|}
\hline \multirow{2}{*}{ Factor } & \multirow{2}{*}{ Medidas descriptivas } & \multicolumn{3}{|c|}{ Titularidad del centro } \\
\hline & & Público & Concertado & Privado \\
\hline \multirow{3}{*}{$\begin{array}{c}\text { A: eficacia en la implicación de } \\
\text { los estudiantes }\end{array}$} & Media & 4,26 & 4,35 & 4,32 \\
\hline & Mediana & 4,25 & 4,25 & 4,25 \\
\hline & Desviación típica & 0,5827 & 0,4808 & 0,4576 \\
\hline \multirow{3}{*}{$\begin{array}{l}\text { B: eficacia en las estrategias de } \\
\text { enseñanza y aprendizaje }\end{array}$} & Media & 4,44 & 4,50 & 4,56 \\
\hline & Mediana & 4,50 & 4,50 & 4,63 \\
\hline & Desviación típica & 0,4901 & 0,4501 & 0,3779 \\
\hline \multirow{3}{*}{ C: eficacia en el manejo de clase } & Media & 4,17 & 4,33 & 4,40 \\
\hline & Mediana & 4,20 & 4,30 & 4,40 \\
\hline & Desviación típica & 0,6623 & 0,4942 & 0,4715 \\
\hline \multirow{3}{*}{$\begin{array}{l}\text { D: eficacia en la atención a la } \\
\text { singularidad de los estudiantes }\end{array}$} & Media & 4,28 & 4,28 & 4,39 \\
\hline & Mediana & 4,25 & 4,25 & 4,50 \\
\hline & Desviación típica & 0,6121 & 0,5312 & 0,4696 \\
\hline \multirow{3}{*}{ GLOBAL } & Media & 4,28 & 4,36 & 4,42 \\
\hline & Mediana & 4,35 & 4,41 & 4,47 \\
\hline & Desviación típica & 0,5028 & 0,4167 & 0,3307 \\
\hline
\end{tabular}




\section{Tabla 3. Estadísticos descriptivos del sentimiento de autoeficacia en los profesores para cada factor, según el nivel educativo}

\begin{tabular}{|c|c|c|c|c|c|}
\hline \multirow{2}{*}{ Factor } & \multirow{2}{*}{ Medidas descriptivas } & \multicolumn{4}{|c|}{ Nivel educativo } \\
\hline & & Infantil & Primaria & Secundaria & Total \\
\hline \multirow{3}{*}{$\begin{array}{c}\text { A: eficacia en la implicación de los } \\
\text { estudiantes }\end{array}$} & Media & 4,38 & 4,33 & 4,27 & 4,31 \\
\hline & Mediana & 4,25 & 4,25 & 4,25 & 4,25 \\
\hline & Desviación típica & 0,5132 & 0,5437 & 0,4953 & 0,52295 \\
\hline \multirow{3}{*}{$\begin{array}{l}\text { B: eficacia en las estrategias de } \\
\text { enseñanza y aprendizaje. }\end{array}$} & Media & 4,52 & 4,45 & 4,52 & 4,48 \\
\hline & Mediana & 4,50 & 4,50 & 4,50 & 4,50 \\
\hline & Desviación típica & 0,4501 & 0,5000 & 0,4014 & 0,4602 \\
\hline \multirow{3}{*}{ C: eficacia en el manejo de clase } & Media & 4,29 & 4,23 & 4,33 & 4,27 \\
\hline & Mediana & 4,4 & 4,3 & 4,2 & 4,20 \\
\hline & Desviación típica & 0,5393 & 0,6229 & 0,4989 & 0,5717 \\
\hline \multirow{3}{*}{$\begin{array}{l}\text { D: eficacia en la atención a la } \\
\text { singularidad de los estudiantes }\end{array}$} & Media & 4,43 & 4,27 & 4,28 & 4,29 \\
\hline & Mediana & 4,25 & 4,25 & 4,25 & 4,25 \\
\hline & Desviación típica & 0,5082 & 0,5705 & 0,5536 & 0,5593 \\
\hline \multirow{3}{*}{ Global } & Media & 4,40 & 4,31 & 4,35 & 4,34 \\
\hline & Mediana & 4,41 & 4,38 & 4,41 & 4,35 \\
\hline & Desviación típica & 0,4117 & 0,4850 & 0,3994 & 0,4472 \\
\hline
\end{tabular}

De acuerdo con la tabla 4, se puede apreciar que el valor-p asociado al estadístico de prueba F es mayor que 0,05 para todas las variables, y es posible concluir, para cada caso, que no hay diferencias significativas en las medias de los correspondientes puntajes de los sentimientos de autoeficacia en la muestra de profesores chilenos, según el nivel educativo en el cual se desempeñen.
Por su parte, la tabla 5 también muestra para la variable Administración del Centro Educativo, que el valor-p en el Factor C (Eficacia en el manejo de clase) es menor a 0,05, lo que confirma evidencia significativa para rechazar la hipótesis de igualdad de medias, a un nivel de significación del $5 \%$. Para corroborar lo anterior, se aplicó el Test de Comparaciones Múltiples de Tuckey Tipo b, el cual arrojó los siguientes subconjuntos homogéneos.

Tabla 4. Análisis de varianza (ANOVA)

\begin{tabular}{|c|c|c|c|c|}
\hline \multirow[t]{2}{*}{ Factores } & \multicolumn{2}{|c|}{ Nivel de enseñanza } & \multicolumn{2}{|c|}{$\begin{array}{l}\text { Administración del } \\
\text { centro }\end{array}$} \\
\hline & $\mathrm{F}$ & Valor-p & $\mathrm{F}$ & Valor-p \\
\hline Factor A: eficacia en la implicación de los estudiantes & 1,178 & 0,309 & 1,195 & 0,304 \\
\hline Factor B: eficacia en las estrategias de enseñanza y aprendizaje & 1,545 & 0,204 & 1,945 & 0,144 \\
\hline Factor C: eficacia en el manejo de clase & 2,124 & 0,121 & 4,469 & 0,012 \\
\hline $\begin{array}{l}\text { Factor D: eficacia en la atención a la singularidad de los } \\
\text { estudiantes }\end{array}$ & 1,680 & 0,187 & 1,166 & 0,312 \\
\hline Global & 0,946 & 0,389 & 2,283 & 0,103 \\
\hline
\end{tabular}


Tabla 5. Test de Tuckey Tipo b

\begin{tabular}{|c|c|c|}
\hline Titularidad del centro & Subconjunto 1 & Subconjunto $\mathbf{2}$ \\
\hline Público & 4,17 & \\
\hline Concertado & 4,33 & 4,33 \\
\hline Privado & & 4,40 \\
\hline
\end{tabular}

Es posible inferir que el puntaje medio para el factor C transformado de los profesores que se desempeñan en centros de administración privada es significativamente mayor que el de los profesores que trabajan en centros públicos. En tanto, no presenta diferencias estadísticamente significativas con los puntajes de los profesores de centros concertados.

\section{Conclusiones}

Con respecto a los resultados obtenidos según el nivel de enseñanza infantil, primario y secundario, los niveles en los sentimientos de autoeficacia en profesores son bastante altos y homogéneos tanto a nivel global como específico del instrumento. No obstante, los profesores del nivel infantil parecen tener índices más altos de autoeficacia que su contraparte de primaria y secundaria, sobrepasándolos en 15 puntos en el factor D ("Eficacia en la atención a la singularidad de los estudiantes"). Así, es posible indicar que los resultados son coincidentes con el estudio de Sari, Çeliköz y Seçer (2009), por cuanto los profesores de enseñanza infantil se sienten mayormente eficaces hacia la enseñanza inclusiva.

No obstante, la comparación de medias entre los sentimientos de autoeficacia y el nivel educativo en el cual se desempeñan los profesores, no ha revelado diferencias significativas. Estos resultados, a la vez que rechazan la hipótesis de investigación que aseveraba diferencias significativas entre ambas variables, son contrarios a los estudios que revelan mayores y mejores sentimientos de autoeficacia para los profesores de enseñanza primaria que para los de enseñanza secundaria (Fives y Buehl, 2010; Wolters y Daugherty, 2007).
Dos posibles explicaciones podrían otorgarse a esta igualdad de sentimientos entre profesores de enseñanza infantil, primaria y secundaria. La primera acerca de la dificultad del instrumento utilizado para capturar lo que realmente ejecutan (o creen ejecutar pero no lo hacen), los profesores en sus aulas, de ahí la homogeneidad en las puntuaciones. La segunda explicación podría estar en la composición de la muestra ( $9,2 \%$ de profesores de infantil y el $38,6 \%$ de profesores de secundaria).

Por lo que se refiere a las medidas descriptivas de los sentimientos de autoeficacia según la titularidad del centro (público, concertado o privado) donde trabajan los profesores, los resultados han revelado que los profesores con desempeño en centros privados presentan una mayor autoeficacia que los que se desempeñan en centros públicos. Pese a lo anterior, el profesorado de centros públicos y concertados ha revelado tener mayores niveles de autoeficacia para el Factor D ("Eficacia en la atención a la singularidad de los estudiantes"), por lo que estarían más habituados a procesos de enseñanza y aprendizaje con grupos de estudiantes heterogéneos.

Con respecto a la comparación de medias entre los sentimientos de autoeficacia y la titularidad de los centros, cabe señalar que no arrojó diferencias significativas para el Puntaje Global del instrumento. Sin embargo, al segmentar factor por factor, los profesores que imparten enseñanza en centros privados albergan sentimientos de autoeficacia significativamente mayores que su contraparte de centros públicos en el factor C ("Eficacia en el manejo de la clase"). Por todo lo anterior, se confirma la hipótesis de investigación sobre la presencia de diferencias significativas entre el sentimiento de autoeficacia y la titularidad de los centros educativos.

A la luz de estos resultados, y de manera similar a lo acontecido con la variable experiencia, la variable titularidad del centro también se encuentra limitada en cuanto a la generalización de los resultados obtenidos. Esto es debido a que los profesores 
que se desempeñan en centros privados solo representan un $11,4 \%$ de la muestra total.

Sumado a lo anterior, y pese a la dificultad de las inferencias, es preciso indicar que los profesores de centros privados han manifestado sentirse más autoeficaces que los de centros públicos para manejar lo que ocurre en sus aulas. Indudablemente, los profesores de centros públicos, al manejar clases con grupos de estudiantes más diversos, deben sortear complejas situaciones de orden y de disciplina, a fin de favorecer y crear un buen clima para el aprendizaje.

Ahora bien, gran parte de los reportes que comparan los sentimientos de autoeficacia docente con la titularidad de los centros donde trabajan los docentes se ha estudiado desde perspectivas socioeconómicas y demográficas. Así, por ejemplo, Takahashi (2011) ha indicado que los centros educativos cuyos estudiantes obtienen resultados mixtos en las pruebas estandarizadas, y provienen de familias con bajos ingresos — que en este caso serían los que convergen en los centros de enseñanza pública— se caracterizan por atraer y retener profesores con bajos niveles de autoeficacia. Por su parte, Siwatu (2011) ha manifestado que los profesores que imparten enseñanza en centros educativos suburbanos, además de poseer mayores sentimientos de autoeficacia para enseñar, creen tener una mejor preparación que los profesores que se desempeñan centros urbanos.

En consecuencia, cabe señalar que para tener una comprensión más completa del comportamiento de la variable utilizada, es relevante indicar que los sentimientos de autoeficacia no constituyen rasgos estables, por el contrario, son fluctuantes y específicos de un contexto específico. De ahí que, la autoeficacia de un docente puede estar condicionada, positiva o negativamente, según el análisis y la evaluación que haga de su labor, independiente del influjo de los estereotipos sociales y culturales que albergan sus escuelas (Bandura, 1997; Knoblauch y Woolfolk-Hoy, 2008; Siwatu, 2011; Tschannen-Moran et al., 1998; Usher y Pajares, 2008).

\section{Referencias}

Armor, D., Conroy-Oseguera P., Cox, M., King, N., Mcdonnell, L., Pascal, A. et al. (1976). Analysis of the school preferred Reading programs in selected Los Angeles minority schools (Inform No. R-2007-LAUSD). Santa Monica: The Rand Corporation.

Bandura, A. (1977). Self-efficacy:Toward a unifying theory of behavioral change. Psychological Review, 84 (2), 191-215.

Bandura, A. (1987). Pensamiento y acción. Fundamentos sociales. Barcelona: Martínez Roca.

Bandura, A. (1997). Self-efficacy: The exercise of control. New York: Freeman.

Bandura, A. (1999). Ejercicio de la eficacia personal y colectiva en sociedades cambiantes. En Bandura, A. (ed.). Autoeficacia. Cómo afrontamos los cambios de la sociedad actual (pp.19-54). Bilbao: Descleé De Brouwer.

Bandura, A. (2004). The growing primacy of perceived efficacy in human self-development, adaptation and change. En Salanova, M., Grau, R., Cifre, E., Llorens, S. y García-Renedo, M. (eds.). Nuevos horizontes en la investigación sobre la autoeficacia (pp. 35-51). Castellón: Universitat Jaume I. Servicio de Comunicación y Publicaciones. 
ISSN $0123-1294$ | e-ISSN 2027-5358 | Educ.Educ. Vol. 19. No.3 | Septiembre-Diciembre de 2016 | pp. 339-354.

Universidad de La Sabana | Facultad de Educación

Bandura, A. (2008). The reconstrual of "free will" from the perspective of social cognitive theory. En Baer, J., Kaufman, J. C. y Baumeister, R. F. (eds.). Are we free? Psychology and free will (pp. 86-127). New York: Oxford University Press.

Berman, P., McLaughlin, M., Bass, G., Pauly, E. y Zellman, G. (1977). Federal Programs supporting educational change: factors affecting implementation and continuation (Inform No. R-1589/7-HEW). Santa Monica: The Rand Corporation (No. de Servicio de reproducción de documentos ERIC ED 140432).

Blanco, Á. (2009). El modelo cognitivo social del desarrollo de la carrera: revisión de más de una década de investigación empírica. Revista de Educación, 350, 423-445.

Bueno, J. A. (2004). La motivación del alumno en el aula. Madrid: ICCE.

Charalambous, C., Philippou, G. y Kyriakides, L. (2008). Tracing the development of preservice teachers' efficacy beliefs in teaching mathematics during fieldwork. Educational Studies in Mathematics, 67 (2), 125-142.

Cheung, H. Y. (2006). The measurement of teacher efficacy: Hong Kong primary in-service teachers. Journal of Education for Teaching, 32 (4), 435-451.

Chong, W., Klassen, R. M., Huan, V. S., Wong, I. y Kates, A. (2010). The relationships among school types, teacher efficacy beliefs, and academic climate: perspective from Asian middle schools. Journal of Educational Research, 103 (3), 183-190.

Ciani, K. D., Summers, J. J.y Easter, M. A. (2008). A “top-down" analysis of high school teacher motivation. Contemporary Educational Psychology, 33 (4), 533-560.

Coladarci, T. y Breton, W. (1997). Teacher efficacy, supervision and the special education resource-room teacher. Journal of Educational Research, 90 (4), 230-239.

De la Torre, M. J. y Casanova, P. F. (2007). Comparative analysis of expectancies of efficacy in in-service and prospective teachers. Teaching and Teacher Education, 23 (5), 641-652.

Dellinger, A. B., Bobbett, J. J., Olivier, D. F. y Ellett, C. D. (2008). Measuring teachers' self-efficacy beliefs: development and use of the TEBS-Self. Teaching and Teacher Education, 24 (2), 751-766.

Fives, H. y Buehl, M. M. (2010). Examining the factor structure of the Teachers' Sense of Efficacy Scale. The Journal of Experimental Education, 78 (1), 118-134.

Friedman, I. y Kass, E. (2002). Teacher self-efficacy: a classroom-organization conceptualization. Teaching and Teacher Education, 18 (6), 675-686.

Gibson, L. S. y Dembo, M. H. (1984). Teacher efficacy: a construct validation. Journal of Educational Psychology, 76 (4), 569-582.

Goddard, R. D. (2001). Collective efficacy: a neglected construct in the study of schools and student achievement. Journal of Educational Psychology, 93 (3), 467-476. 
Guo, Y., Justice, L., Sawyer, B. y Tompkins, V. (2011). Exploring factors related to Preschool teachers' self-efficacy. Teaching and Teacher Education, 27 (5), 961-968.

Guskey, T. R. y Passaro, P. (1994). Teacher efficacy: A study of construct dimensions. American Educational Research Journal, 31 (3), 627-643.

Hernández, R., Fernández, C.y Baptista, P. (2010). Fundamentos de metodología de la investigación (2 ed.). Madrid: McGraw-Hill.

Klassen, R. M., Tze, V. M. C., Betts, S. M.y Gordon, K. A. (2011). Teacher efficacy research 1998-2009: signs of progress or unfulfilled promise? Educational Psychological Review, 23 (1), 21-43.

Knoblauch, D. y Woolfolk-Hoy, A. (2008). Maybe I can teach those kids. The influence of contextual factors on student teachers' efficacy beliefs. Teaching and Teacher Education, 24 (1), 166-179.

Martínez, R., Moreno, R.y Muñiz, J. (2005). Construcción de los ítems. En Muñiz, J., Fidalgo, A., García Cueto, E., Martínez, R. y Moreno, R. (eds.). Cuadernos de Estadística 3o. Análisis de los ítems (pp. 9-52). Madrid: La Muralla.

Pajares, F. (2006). Self-efficacy during childhood and adolescence. En Pajares, F.y Urdan, T. (eds.). Adolescence and education. Self-efficacy beliefs of adolescents. Greenwich: Information Age Publishing.

Pas, E. T., Bradshaw, C. P.y Hershfeldt, P. A. (2012). Teacher- and school-level predictors of teacher efficacy and burnout: identifying potential areas for support. Journal of School Psychology, 50 (1), 129-145.

Philippou, G. N. y Christou, C. (2002). A study of mathematics teaching efficacy beliefs of Primary teachers. En Leder, G., Pehkonen, E. y Toerner, G. (eds.). Beliefs: a hidden variable in mathematics education? (pp. 211-232). Dordrecht: Kluwer.

Pintrich, P. R. y Schunk, D. H. (2006). Motivación en contextos educativos: teoría, investigación y aplicaciones. Madrid: Pearson-Educación.

Prieto, L. (2007). Autoeficacia del profesor universitario. Eficacia percibida y práctica docente. Madrid: Narcea.

Rizvi, M. y Elliot, B. (2005). Teachers' perceptions of their professionalism in government Primary schools in Karachi, Pakistan. Asia Pacific Journal of Teacher Education, 33 (1), 35-52.

Ross, J.y Bruce, C. (2007). Professional development effects on teacher efficacy: results of randomized field trial. Journal of Educational Research, 101 (1), 50-60.

Sari, H., Çeliköz, N.y Seçer, Z. (2009). An analysis of pre-school teachers' and student teachers' attitudes to inclusion and their self-efficacy. International Journal of Special Education, 24 (3), 29-44.

Siwatu, K. O. (2011). Preservice teachers' sense of preparedness and self-efficacy to teach in America's urban and suburban schools: Does context matter? Teaching and Teacher Education, 27 (2), 357-365.

Soodak, L.y Podell, D. (1996). Teacher efficacy: Toward the understanding of a multifaceted construct. Teaching and Teacher Education, 12 (4), 401-411. 
ISSN $0123-1294$ | e-ISSN 2027-5358 | Educ.Educ. Vol. 19. No.3 | Septiembre-Diciembre de 2016 | pp. 339-354.

Universidad de La Sabana | Facultad de Educación

Soodak, L.y Podell, D. M. (1997). Efficacy and experience: perceptions of efficacy among preservice and practicing teachers. Journal of Research and Development in Education, 30 (4), 214-221.

Takahashi, S. (2011). Co-constructing efficacy: A “communities of practice” perspective on teachers' efficacy beliefs. Teaching and Teacher Education, 27 (4), 732-741.

Tschannen-Moran, M. y Woolfolk, A. (2001). Teacher efficacy: capturing an elusive construct. Teaching and Teacher Education, 17 (7), 783-805.

Tschannen-Moran, M. y Woolfolk, A. (2007). The differential antecedents of self efficacy beliefs of novice and experienced teachers. Teaching and Teacher Education, 23 (6), 944-956.

Tschannen-Moran, M., Woolfolk, A. y Hoy, W. K. (1998). Teacher efficacy: Its meaning and measure. Review of Educational Research, 68 (2), 202-248.

Usher, E. y Pajares, F. (2008). Sources of self-efficacy in school: critical review of the literature and future directions. Review of Educational Research, 78 (4), 751-796.

Wolters, C. A. y Daugherty, S. G. (2007). Goal Structures and Teachers' Sense of Efficacy: Their Relation and Association to Teaching Experience and Academic Level. Journal of Educational Psychology, 99 (1), 181-193. 